\title{
Classical State Masking over a Quantum Channel
}

\author{
Uzi Pereg, ${ }^{1,2, *}$ Christian Deppe ${ }^{1, \dagger}$ and Holger Boche ${ }^{3,2,4, \ddagger}$ \\ ${ }^{1}$ Institute for Communications Engineering, Technical University of Munich \\ ${ }^{2}$ Munich Center for Quantum Science and Technology (MCQST) \\ ${ }^{3}$ Theoretical Information Technology, Technical University of Munich \\ ${ }^{4}$ Cyber Security in the Age of Large-Scale Adversaries Exzellenzcluster (CASA)
}

\begin{abstract}
Transmission of classical information over a quantum state-dependent channel is considered, when the encoder can measure channel side information (CSI) and is required to mask information on the quantum channel state from the decoder. In this quantum setting, it is essential to conceal the CSI measurement as well. A regularized formula is derived for the masking equivocation region, and a full characterization is established for a class of measurement channels.
\end{abstract}

\section{INTRODUCTION}

Security and privacy are critical aspects in modern communication systems [1-4]. In Wyner's wiretap setting [5], the sender transmits a sequence $X^{n}$ over a memoryless broadcast channel $p_{Y, Z \mid X}$, such that the output sequence $Y^{n}$ is decoded by the legitimate receiver, while $Z^{n}$ is received by a malicious eavesdropper. Confidentiality requires that the eavesdropper cannot obtain information on the transmitted message from the sequence $Z^{n}$. On the other hand, Merhav and Shamai [6] introduced a communication system with the privacy requirement of masking.

In the classical masking setting, the sender transmits a sequence $X^{n}$ over a memoryless state-dependent channel $p_{Y \mid X, S}$, where the state sequence $S^{n}$ has a fixed memoryless distribution and is not affected by the transmission. The transmitter of $X^{n}$ is informed of $S^{n}$ and is required to send information to the receiver while limiting the amount of information that the receiver can learn about $S^{n}$. Intuitively, as the transmitter uses the side information in order to increase the transmission rate, more information on the channel state may be revealed. Hence, there is a tradeoff between high transmission rate and low leakage of information [6]. The masking setting can also be viewed as communication with an untrusted party, where Alice wishes to send Bob a limited amount of information, while keeping the information source hidden [7-11]. It is expected that protocols that can solve communication tasks even under untrusted hardware platforms or untrusted software implementations will play an important role in the development of future communication systems [12,13]. Related settings and extensions are also considered in [14-21].

Quantum information technology is rapidly evolving in both practice and theory [22]. Communication through quantum channels can be separated into different categories. In particular, in quantum information theory and Shannon theory, the following models of communication over quantum channels are considered in the literature:

A) Transmission of classical information without assistance.

B) Subspace transmission without assistance.

C) Communication with entanglement assistance.

For classical communication without assistance, model A, the Holevo-Schumacher-Westmo- reland (HSW) Theorem provides a regularized ("multi-letter") formula for the capacity of a quantum channel [23, 24]. Although calculation of such a formula is intractable in general, it provides computable lower bounds, and there are special cases where the capacity can be computed exactly $[25,26]$. The reason for this difficulty is that the Holevo information is not necessarily additive [26]. A similar difficulty occurs in model B, treating the transmission of quantum information [27].

Model $\mathrm{C}$ above is a scenario where Alice and Bob have access to entanglement resources that are shared a priori, before communication takes place. While entanglement can be used to produce shared randomness, it is a much more powerful aid [28]. E.g., using super-dense coding, entanglement assistance doubles the transmission rate of classical messages over a noiseless qubit channel. The entanglement-assisted capacity of a noisy quantum channel was fully characterized by Bennett et al. [29] in terms of the quantum mutual information. Entanglement resources are thus instrumental for the analysis of quantum communication systems, providing a computable upper bound for unassisted communication as well.

\footnotetext{
* uzi.pereg@tum.de

$\dagger$ christian.deppe@tum.de

$\ddagger$ boche@tum.de
} 


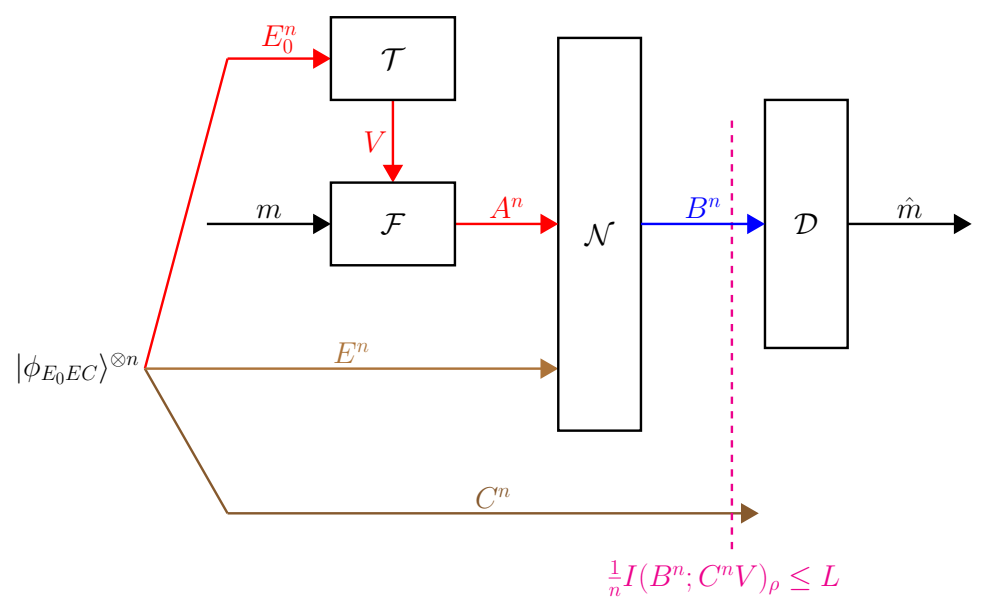

FIG. 1. Coding for a quantum state-dependent channel $\mathcal{N}_{E A \rightarrow B}$ given side information at the encoder and masking from the decoder. The quantum systems of Alice and Bob are marked in red and blue, respectively. The channel state systems $E^{n}$ and $C^{n}$ are marked in brown. Alice wishes to send a classical message $m$ to Bob. She has access to side-information systems $E_{0}^{n}$, which are entangled with the channel state systems $E^{n}$. Alice performs a measurement $\mathcal{T}$, and obtains a measurement outcome $V$. Then, she applies an encoding map $\mathcal{F}:(m, V) \rightarrow \rho_{A^{n}}$, and transmits the system $A^{n}$ over the channel. Bob receives the channel output system $B^{n}$, and applies the decoding measurement $\mathcal{D}: \rho_{B^{n}} \rightarrow \hat{m}$ to obtain an estimate $\hat{m}$ for Alice's message, as a measurement outcome. A leakage rate $L$ is achieved if $\frac{1}{n} I\left(B^{n} ; C^{n} V\right)_{\rho} \leq L$.

Boche, Cai, and Nötzel [30] addressed classical-quantum channels with channel side information (CSI) at the encoder. The capacity was determined given causal CSI, and a regularized formula was provided given non-causal CSI [30]. The first author [31,32] extended the results to a quantum-input quantum-output channel with random parameters, and further considered communication over quantum channels with parameter estimation at the receiver, given either strictly-causal, causal, or non-causal CSI at the encoder, and without CSI as well. Warsi and Coon [33] used an information-spectrum approach to derive multi-letter bounds for a similar setting with rate-limited CSI. The entanglement-assisted capacity of a quantum channel with non-causal CSI was determined by Dupuis in [34, 35] (see also [36]), and with causal CSI in [37, 38]. Luo and Devetak [39] considered channel simulation with source side information (SSI) at the decoder, and also solved the quantum generalization of the Wyner-Ziv problem [40]. Quantum data compression with SSI is also studied in [41-47].

Considering secure communication over the quantum wiretap channel, Devetak [27] and Cai et al. [48] established a regularized characterization of the secrecy capacity without assistance. Related models appear in [49-54] as well. Boche et al. $[55,56]$ studied the quantum wiretap channel with an active jammer. The capacity-equivocation region, characterizing the tradeoff between secret key consumption and private classical communication, was established in $[49,51]$. The quantum Gel'fand-Pinsker wiretap channel is considered in [54] and other related scenarios can be found in [57-59]. Furthermore, network settings with confidential messages were recently considered in [60-62], respectively.

In quantum channel state masking, analogously to the classical model [6], the channel state system $C$ store undesired quantum information which leaks to the receiver. This can model a leakage of private network information to the end-user. Alternatively, $C^{n}$ may represent a separate transmission to another receiver (Charlie), in a product state, out of our control, and which is not intended to our receiver (Bob), and is therefore to be concealed from him. Thus, the goal of the transmitter (Alice) is to mask this undesired information as much as possible on the one hand, and to transmit reliable information on the other. Masking can also be viewed as a building block for cryptographic problems of oblivious transfer of information and secure computation by untrusting parties. In a recent paper by the authors [63], we considered a quantum state-dependent channel, when the encoder has CSI and is required to mask information on the quantum channel state from the decoder. We have established a full characterization for the entanglement-assisted masking region with maximally correlated channel state systems, and a regularized formula for the quantum masking region without assistance. That is, we addressed model $\mathrm{B}$ and model $\mathrm{C}$ for quantum channel state masking in [63].

In this paper, we consider model A of a quantum state-dependent channel $\mathcal{N}_{E A \rightarrow B}$, when the encoder has CSI and is required to mask information on the quantum channel state from the decoder. We derive a regularized formula for the classical masking region and establish full characterization for a class of measurement channels. Here, however, 
the communication task is to send classical information, while there are no entanglement resources available to Alice and Bob. Specifically, the channel state systems are in an entangled state $\left|\phi_{E_{0} E C}\right\rangle^{\otimes n}$. Alice wishes to send a classical message $m$. To this end, she measures the CSI systems $E_{0}^{n}$ and obtains an outcome $V$. Based on the measurement outcome, Alice encodes the quantum state of the channel input systems $A^{n}$ in such a manner that limits the leakagerate of Bob's information on $C^{n}$ from $B^{n}$, while the systems $E_{0}^{n}$ and $C^{n}$ are entangled with the channel state systems $E^{n}$ (see Figure 1).

The quantum model involves three channel state systems, $E^{n}, E_{0}^{n}$, and $C^{n}$, as opposed to the classical case [6] of a single random parameter. The system $E_{0}^{n}$ can be thought of as part of the environment of both our transmitter and the source of $C^{n}$, possibly entangled if they had previous interaction, while $E^{n}$ belongs to the channel's environment. The interpretation given in [35], for the entanglement between $E_{0}^{n}$ and $E^{n}$, is that Alice shares entanglement with the channel itself. Another distinction from the classical case is that the measurement can cause a collapse of the wave function, hence correlations can be lost. Thereby, it is essential to conceal the CSI observation as well. In the present model, the leakage requirement involves both the masked system $C^{n}$ and the measurement outcome $V$. Those subtleties do not exist in the classical problem.

Compared to our previous work [63], we now address a more fundamental problem in the following sense. In model A, we consider a classical task, i.e. the transmission of classical bits, that is performed using a quantum apparatus. The techniques in the analysis are significantly different as well. The proof in [63] is based on the decoupling approach [64], using a code that decouples both Bob's environment and the channel state systems from the input reference. Here, the analysis is based on the quantum packing lemma [65], using type-class projectors and the classical binning technique, along with non-trivial arguments to establish the leakage requirement.

\section{DEFINITIONS AND RELATED WORK}

\section{A. Notation, States, and Information Measures}

We use the following notation conventions. Calligraphic letters $\mathcal{X}, \mathcal{Y}, \mathcal{Z}, \ldots$ are used for finite sets. Lowercase letters $x, y, z, \ldots$ represent constants and values of classical random variables, and uppercase letters $X, Y, Z, \ldots$ represent classical random variables. The distribution of a random variable $X$ is specified by a probability mass function (pmf) $p_{X}(x)$ over a finite set $\mathcal{X}$. We use $x^{j}=\left(x_{1}, x_{2}, \ldots, x_{j}\right)$ to denote a sequence of letters from $\mathcal{X}$. A random sequence $X^{n}$ and its distribution $p_{X^{n}}\left(x^{n}\right)$ are defined accordingly.

The state of a quantum system $A$ is given by a density operator $\rho$ on the Hilbert space $\mathcal{H}_{A}$. The state is said to be pure if $\rho=|\psi\rangle\langle\psi|$, for some vector $|\psi\rangle \in \mathcal{H}_{A}$, where $\langle\psi|$ is the Hermitian conjugate of $|\psi\rangle$. A measurement of a quantum system is any set of operators $\left\{\Lambda_{j}\right\}$ that forms a positive operator-valued measure (POVM), i.e. the operators are positive semi-definite and $\sum_{j} \Lambda_{j}=\mathbb{1}$, where $\mathbb{1}$ is the identity operator. According to the Born rule, if the system is in state $\rho$, then the probability of the measurement outcome $j$ is given by $p_{A}(j)=\operatorname{Tr}\left(\Lambda_{j} \rho\right)$.

Define the quantum entropy of the density operator $\rho$ as

$$
H(\rho) \triangleq-\operatorname{Tr}[\rho \log (\rho)] .
$$

We may also consider the state of a pair of systems $A$ and $B$ on the tensor product $\mathcal{H}_{A} \otimes \mathcal{H}_{B}$ of the corresponding Hilbert spaces. Given a bipartite state $\rho_{A B}$, define the quantum mutual information as

$$
I(A ; B)_{\rho}=H\left(\rho_{A}\right)+H\left(\rho_{B}\right)-H\left(\rho_{A B}\right) .
$$

Furthermore, the conditional quantum entropy and mutual information are defined by $H(A \mid B)_{\rho}=H\left(\rho_{A B}\right)-H\left(\rho_{B}\right)$ and $I(A ; B \mid C)_{\rho}=H(A \mid C)_{\rho}+H(B \mid C)_{\rho}-H(A, B \mid C)_{\rho}$, respectively.

A pure bipartite state is called entangled if it cannot be expressed as the tensor product of two states in $\mathcal{H}_{A}$ and $\mathcal{H}_{B}$. The maximally entangled state between two systems of dimension $D$ is defined by $\left|\Phi_{A B}\right\rangle=\frac{1}{\sqrt{D}} \sum_{j=0}^{D-1}|j\rangle_{A} \otimes$ $|j\rangle_{B}$, where $\left\{|j\rangle_{A}\right\}_{j=0}^{D-1}$ and $\left\{|j\rangle_{B}\right\}_{j=0}^{D-1}$ are respective orthonormal bases. Note that $I(A ; B)_{|\Phi\rangle\langle\Phi|}=2 \cdot \log (D)$ and $I(A\rangle B)_{|\Phi\rangle\langle\Phi|}=\log (D)$.

\section{B. Quantum Channel}

A quantum channel maps a quantum state at the sender system to a quantum state at the receiver system. Here, we consider a channel with two inputs, where one of the inputs, which is referred to as the channel state, is not controlled by the encoder. Formally, a quantum state-dependent channel $\left(\mathcal{N}_{E A \rightarrow B},\left|\phi_{E E_{0} C}\right\rangle\right)$ is defined by a linear, 
completely positive, trace preserving map $\mathcal{N}_{E A \rightarrow B}$ and a quantum state $\left|\phi_{E E_{0} C}\right\rangle$. This model can be interpreted as if the channel is entangled with the systems $E, E_{0}$, and $C$.

We assume that both the channel state systems and the quantum channel have a product form. That is, the joint state of the systems $E^{n}=\left(E_{1}, \ldots, E_{n}\right), E_{0}^{n}=\left(E_{0,1}, \ldots, E_{0, n}\right)$ and $C^{n}=\left(C_{1}, \ldots, C_{n}\right)$ is $\left|\phi_{E E_{0} C}\right\rangle^{\otimes n}$, and if the systems $A^{n}=\left(A_{1}^{\prime}, \ldots, A_{n}^{\prime}\right)$ are sent through $n$ channel uses, then the input state $\rho_{E^{n}} A^{n}$ undergoes the tensor product mapping $\mathcal{N}_{E^{n} A^{n} \rightarrow B^{n}} \equiv \mathcal{N}_{E A \rightarrow B}^{\otimes n}$. Given CSI, the transmitter can measure the systems $E_{0}^{n}$, which are entangled with the channel state systems $E^{n}$. We will further consider a secrecy requirement that limits the information that the receiver can obtain on $C^{n}$. The sender and the receiver are often referred to as Alice and Bob.

Remark 1. Our results apply to the case where $E, E_{0}$, and $C$ are in a mixed state as well. Specifically, given a mixed state $\varphi_{E E_{0} C}$, there exists a purification $\left|\phi_{G E E_{0} C}\right\rangle$, such that the reduced density operator for this purification is $\varphi_{E E_{0} C}$. Hence, we can redefine the channel as follows. First, replace the channel state system $E$ by $\tilde{E}=(G, E)$, and then consider the quantum state-dependent channel $\widetilde{\mathcal{N}}_{\tilde{E} A \rightarrow B}$, where

$$
\widetilde{\mathcal{N}}_{G E A \rightarrow B}\left(\rho_{G E A}\right)=\mathcal{N}_{E A \rightarrow B}\left(\operatorname{Tr}_{G}\left(\rho_{G E A}\right)\right) \text {. }
$$

We will also consider the quantum-classical special case.

Definition 1. A measurement channel (or, q-c channel) $\mathcal{M}_{A \rightarrow Y}$ has the following form,

$$
\mathcal{M}_{A \rightarrow Y}\left(\rho_{A}\right)=\sum_{y \in \mathcal{Y}} \operatorname{Tr}\left(\Lambda_{y} \rho_{A}\right)|y\rangle\langle y|
$$

for some POVM $\left\{\Lambda_{y}\right\}$ and orthonormal vectors $\{|y\rangle\}$. In order to distinguish it from the general channel, we denote the state-dependent measurement channel by $\left(\mathcal{M}_{E A \rightarrow Y},|\phi\rangle\right)$.

One may also consider the special case where the channel state is fully described by a classical random parameter, i.e. $E \equiv E_{0} \equiv C \equiv S$ where $S \sim q(s)$ is a classical random variable. In this case, the channel can be viewed as a random selection from a collection of channels $\left\{\mathcal{N}_{A \rightarrow B}^{(s)}\right\}_{s \in \mathcal{S}}$. This family of quantum state-dependent channels is of particular interest as it captures the notion of channel uncertainty. For the so-called 'random-parameter quantum channel', the availability of CSI at the encoder simply means that Alice knows the value of $S$. We give simple examples below.

Example 1. The random-parameter depolarizing channel is defined as follows [32, Example 3$]$. Let $\mathcal{N}_{S A \rightarrow B}$ be a quantum state-dependent channel that depends on a classical random parameter $S \in\{0,1,2,3\}$, hence $E_{0} \equiv E \equiv$ $C \equiv S$. As pointed out above, such a random-parameter quantum channel can be viewed as a random selection from a set of channels, $\left\{\mathcal{N}^{(s)}\right\}_{s=0,1,2,3 \text {. Let }}$

$$
\begin{aligned}
& \mathcal{N}^{(0)}(\rho)=\rho \\
& \mathcal{N}^{(1)}(\rho)=\mathrm{X} \rho \mathbf{X} \\
& \mathcal{N}^{(2)}(\rho)=\mathrm{Y} \rho \mathrm{Y} \\
& \mathcal{N}^{(3)}(\rho)=\mathrm{Z} \rho \mathbf{Z}
\end{aligned}
$$

with the following parameter distribution,

$$
q(0)=1-\frac{3 \varepsilon}{4}, q(1)=q(2)=q(3)=\frac{\varepsilon}{4}
$$

where $\varepsilon \in(0,1]$ is a given constant and X, Y, Z are the qubit Pauli operators. In other words, the parameter $S_{i}$ chooses a Pauli operator that is applied to the $i$ th input system. We note that without CSI, the average channel is the same as the standard depolarizing channel, i.e.

$$
\begin{aligned}
\overline{\mathcal{N}}_{A \rightarrow B}(\rho) & \equiv \sum_{s} q(s) \mathcal{N}^{(s)}(\rho) \\
& =\left(1-\frac{3 \varepsilon}{4}\right) \rho+\frac{\varepsilon}{4}(\mathrm{X} \rho \mathbf{X}+\mathrm{Y} \rho \mathbf{Y}+\mathrm{Z} \rho \mathbf{Z}) \\
& =(1-\varepsilon) \rho+\varepsilon \pi
\end{aligned}
$$

where $\pi=\frac{1}{2}$ is the maximally mixed state (see [66, Section 4.7.4]). Without CSI, the capacity can be significantly lower than 1 . In particular, for $\varepsilon=1$, the capacity without CSI is zero and Alice cannot send any information to Bob. 
Knowing the parameter $s$, Alice can revert the operation of the channel by applying the corresponding Pauil operator. That is, Alice applies $\mathcal{N}^{(s)}$ locally in her encoding operation, and then sends the input state $\mathcal{N}^{(s)}(\rho)$ through the channel. Hence, Bob receives $\mathcal{N}^{(s)}\left(\mathcal{N}^{(s)}(\rho)\right)=\rho$. In this manner, we effectively have a noiseless channel. Furthermore, the channel output has no correlation with the channel state $S$. Thereby, Alice can send 1 information bit per transmission without leakage.

Example 2. Consider a random-parameter qubit channel that depends on a classical random parameter $S \sim$ $\operatorname{Bernoulli}(\varepsilon)$, such that

$$
\begin{aligned}
& \mathcal{N}^{(0)}(\rho)=\rho \\
& \mathcal{N}^{(1)}(\rho)=|\psi\rangle\langle\psi|
\end{aligned}
$$

where $|\psi\rangle$ is a given state in the same qubit space. We will return to this example in the sequel and show that if Alice uses the CSI in order to increase the transmission rate, then there may be leakage of information on $S^{n}$ to Bob (see Example 3).

\section{Coding}

We define a privacy masking code to transmit classical information over a quantum channel. With non-causal CSI, Alice can measure the systems $E_{0}^{n}$, which are entangled with the channel state systems $C^{n} E^{n}$. We refer to $E_{0}^{n}$ as the CSI systems.

Definition 2. A $\left(2^{n R}, n\right)$ classical masking code with CSI at the encoder consists of the following: A message set $\left[1: 2^{n R}\right]$, assuming that $2^{n R}$ is integer, an encoding POVM, $\mathcal{T} \equiv\left\{T_{E_{0}^{n}}^{v}\right\}$, on the CSI system $E_{0}^{n}$, an encoding map $\mathcal{F}:(m, v) \mapsto \rho_{A^{n}}$, and a decoding POVM $\mathcal{D} \equiv\left\{D_{B^{n}}^{\hat{m}}\right\}$.

The communication scheme is depicted in Figure 1. The sender Alice has the systems $E_{0}^{n}$ and $A^{n}$, and the receiver Bob has the systems $B^{n}$. Alice chooses a classical message $m \in\left[1: 2^{n R}\right]$ uniformly at random, and wishes to send it to Bob. To this end, she measures the CSI systems $E_{0}^{n}$, which are entangled with the channel state systems, using the measurement set $\mathcal{T}$, and obtains a measurement outcome $v$. Then, Alice encodes the classical message $m$ using the measurement outcome, and prepares the input state $\rho_{A^{n}}^{m, v}=\mathcal{F}(m, v)$. The average post-measurement input state is

$$
\bar{\rho}_{C^{n} E^{n} V A^{n}}^{m}=\sum_{v} \operatorname{Tr}_{E_{0}^{n}}\left(T_{E_{0}^{n}}^{v} \phi_{C E E_{0}}^{\otimes n}\right) \otimes|v\rangle\langle v| \otimes \rho_{A^{n}}^{m, v}
$$

where $V$ is a classical register that stores the CSI-measurement outcome.

Alice transmits the systems $A^{n}$ over $n$ channel uses of $\mathcal{N}_{E A \rightarrow B}$. Hence, the average output state is

$$
\rho_{C^{n} V B^{n}}^{m}=\mathcal{N}_{E^{n} A^{n} \rightarrow B^{n}}\left(\bar{\rho}_{C^{n} V E^{n} A^{n}}^{m}\right) .
$$

Bob receives the channel output and applies the decoding measurement $\mathcal{D}$ to the output systems $B^{n}$, such that the measurement outcome $\hat{m}$ is an estimate of the original message $m$. The average probability of error is

$$
P_{e}^{(n)}(\mathcal{T}, \mathcal{F}, \mathcal{D})=1-\frac{1}{2^{n R}} \sum_{m=1}^{2^{n R}} \operatorname{Tr}\left(D_{B^{n}}^{m} \rho_{B^{n}}^{m}\right)
$$

where $\rho_{B^{n}}^{m}=\operatorname{Tr}_{C^{n} V}\left(\rho_{C^{n} V B^{n}}^{m}\right)$. The masking leakage rate of the code $(\mathcal{T}, \mathcal{F}, \mathcal{D})$ is defined as

$$
\ell^{(n)}(\mathcal{T}, \mathcal{F}, \mathcal{D}) \triangleq \frac{1}{n} I\left(C^{n} V ; B^{n}\right)_{\rho}
$$

where the mutual information is computed with respect to the average states, corresponding to a uniformly distributed message and the random outcome $V$ of the CSI measurement at the encoder. A $\left(2^{n R}, n, \varepsilon, L\right)$ masking code satisfies $P_{e}^{(n)}(\mathcal{T}, \mathcal{F}, \mathcal{D}) \leq \varepsilon$ and $\ell^{(n)}(\mathcal{T}, \mathcal{F}, \mathcal{D}) \leq L$. A rate-leakage pair $(R, L)$, where $R, L \geq 0$, is called achievable if for every $\varepsilon, \delta>0$ and sufficiently large $n$, there exists a $\left(2^{n R}, n, \varepsilon, L+\delta\right)$ masking code.

The classical masking region $\mathbb{R}_{\mathrm{CL}}(\mathcal{N})$ of the quantum state-dependent channel $\mathcal{N}_{E A \rightarrow B}$ is defined as the set of achievable pairs $(R, L)$ with CSI at the encoder. Alternatively, one may fix the leakage rate and consider the optimal transmission rate. The classical capacity-leakage function $\mathbb{C}_{\mathrm{Cl}}(\mathcal{N}, L)$ is defined as the supremum of achievable rates $R$ for a given leakage $L$. Note that $\mathbb{C}_{\mathrm{Cl}}(\mathcal{N}, \infty)$ reduces to the standard definition of the classical capacity of a quantum channel, without a masking requirement.

Remark 2. Observe that if $L \geq 2 \log \left|\mathcal{H}_{B}\right|$, then the masking requirement trivially holds because $I\left(C^{n} V ; B^{n}\right)_{\rho} \leq$ $2 H\left(B^{n}\right)_{\rho} \leq 2 n \log \left|\mathcal{H}_{B}\right|$. That is, if $L \geq 2 \log \left|\mathcal{H}_{B}\right|$, then $\mathbb{C}_{\mathrm{Cl}}(\mathcal{N}, L)=\mathbb{C}_{\mathrm{Cl}}(\mathcal{N}, \infty)$. 


\section{Related Work}

We briefly review known results for the case where there is no masking requirement. First, consider a quantum channel which is not affected by a channel state, i.e. $\mathcal{N}_{E A \rightarrow B}\left(\rho_{E A}\right)=\mathcal{P}_{A \rightarrow B}\left(\operatorname{Tr}_{E}\left(\rho_{E A}\right)\right)$. Define

$$
\chi(\mathcal{P}) \triangleq \max _{p_{X}(x),\left|\phi_{A}^{x}\right\rangle} I(X ; B)_{\rho}
$$

with $\rho_{X B} \equiv \sum_{x \in \mathcal{X}} p_{X}(x)|x\rangle\langle x| \otimes \mathcal{P}\left(\left|\phi_{A}^{x}\right\rangle\left\langle\phi_{A}^{x}\right|\right)$ and $|\mathcal{X}| \leq\left|\mathcal{H}_{A}\right|^{2}$. The objective functional $I(X ; B)_{\rho}$ is referred to as the Holevo information with respect to the ensemble $\left\{p_{X}(x), \mathcal{E}\left(\left|\phi_{A}^{x}\right\rangle\left\langle\phi_{A}^{x}\right|\right)\right\}$ and the channel $\mathcal{P}_{A \rightarrow B}$, while the formula $\chi(\mathcal{P})$ itself is sometimes referred to as the Holevo information of the channel [66]. Next, we cite the HSW Theorem, which provides a regularized capacity formula for a quantum channel that does not depend on a state.

Theorem 1 (see $[23,24]$ ). The classical capacity of a quantum channel $\mathcal{P}_{A \rightarrow B}$ that does not depend on a channel state, without a masking requirement, is given by

$$
\mathbb{C}_{\mathrm{Cl}}(\mathcal{P}, \infty)=\lim _{n \rightarrow \infty} \frac{1}{n} \chi\left(\mathcal{P}^{\otimes n}\right)
$$

A single-letter characterization is an open problem for a general quantum channel. Although calculation of a regularized formula is intractable in general, it provides a computable lower bound, and there are special cases where the capacity can be computed exactly [67].

Next, we move to a quantum state-dependent channel with CSI at the encoder, in the special case where the state is a classical random parameter $S \sim q(s)$. As explained in Subsection II B, the channel $\mathcal{N}_{S A \rightarrow B}$ can be specified by a collection of channels $\left\{\mathcal{N}_{A \rightarrow B}^{(s)}\right\}$. Define

$$
\mathrm{R}(\mathcal{N}, \infty) \triangleq \sup _{p_{X \mid S}(x \mid s), \varphi_{A}^{x}}\left[I(X ; B)_{\rho}-I(X ; S)\right]
$$

where the supremum is over the conditional distributions $p_{X \mid S}$ and the collections of input states $\varphi_{A}^{x}$, such that given $S=s$, we have the state $\rho_{X B \mid s} \equiv \sum_{x \in \mathcal{X}} p_{X \mid S}(x \mid s)|x\rangle\langle x| \otimes \mathcal{N}_{A \rightarrow B}^{(s)}\left(\varphi_{A}^{x}\right)$.

Theorem 2 (see [31, 32]). The classical capacity of a random-parameter quantum channel $\left(\mathcal{N}_{S A \rightarrow B}, S \sim q(s)\right)$, with CSI at the encoder and without a masking requirement, is given by

$$
\mathbb{C}_{\mathrm{Cl}}(\mathcal{N}, \infty)=\lim _{n \rightarrow \infty} \frac{1}{n} \mathrm{R}\left(\mathcal{N}^{\otimes n}, \infty\right) .
$$

\section{MAIN RESULTS}

We state our results on the quantum state-dependent channel $\mathcal{N}_{E A \rightarrow B}$ with masking. We determine a regularized characterization of the masking region and capacity-leakage function, for the transmission of classical information. Define

$$
\mathcal{R}_{\mathrm{Cl}}(\mathcal{N})=\bigcup_{\Lambda_{E_{0}}^{s}, p_{X \mid S}, \varphi_{A}^{x}}\left\{\begin{aligned}
(R, L): 0 \leq R & \leq I(X ; B)_{\rho}-I(X ; S) \\
L & \geq I(C S ; X B)_{\rho}
\end{aligned}\right\}
$$

where the union is over the POVMs $\left\{\Lambda_{E_{0}}^{s}\right\}$, the conditional distributions $p_{X \mid S}$, and the collections of input states $\varphi_{A}^{x}$, with

$$
\rho_{E C S X A}=\sum_{s \in \mathcal{S}} \sum_{x \in \mathcal{X}} p_{X \mid S}(x \mid s) \operatorname{Tr}_{E_{0}}\left(\Lambda_{E_{0}}^{s} \phi_{E_{0} E C}\right) \otimes|s, x\rangle\langle s, x| \otimes \varphi_{A}^{x}
$$

and

$$
\rho_{B C S X}=\mathcal{N}_{E A \rightarrow B}\left(\rho_{E A C S X}\right)
$$

Theorem 3. 
1) The classical masking region of a quantum state-dependent channel $\left(\mathcal{N}_{E A \rightarrow B},\left|\phi_{E E_{0} C}\right\rangle\right)$ with CSI at the encoder is given by

$$
\mathbb{R}_{\mathrm{Cl}}(\mathcal{N})=\bigcup_{n=1}^{\infty} \frac{1}{n} \mathcal{R}_{\mathrm{Cl}}\left(\mathcal{N}^{\otimes n}\right)
$$

2) For a measurement channel $\mathcal{M}_{E A \rightarrow Y}$ with a classical CSI system $E_{0} \equiv S$,

$$
\mathbb{R}_{\mathrm{Cl}}(\mathcal{M})=\bigcup_{p_{X \mid S}, \varphi_{A}^{x}}\left\{\begin{aligned}
(R, L): 0 \leq R & \leq I(X ; Y)-I(X ; S) \\
L & \geq I(C S ; X Y)_{\rho}
\end{aligned}\right\} .
$$

The proof of Theorem 3 is given in Appendix C.

Remark 3. In Appendix A, we show that the union can be exhausted with cardinality $|\mathcal{X}| \leq\left(\left|\mathcal{H}_{A}\right|^{2}+1\right)\left|\mathcal{H}_{E}\right|$. Hence, in principle, the region $\mathcal{R}_{\mathrm{Cl}}(\mathcal{N})$ is computable. Nevertheless, for a general quantum channel, we have only obtained a regularized characterization. As mentioned in Section IID, a single-letter capacity formula is an open problem, even for a point-to-point quantum channel without a channel state.

Equivalently, we can characterize the capacity-leakage function. The following corollary is an immediate consequence of Theorem 3.

\section{Corollary 4.}

1) The classical capacity-leakage function of a quantum state-dependent channel $\left(\mathcal{N}_{E A \rightarrow B},\left|\phi_{E E_{0} C}\right\rangle\right)$ with CSI at the encoder is given by

$$
\mathbb{C}_{\mathrm{Cl}}(\mathcal{N}, L)=\lim _{n \rightarrow \infty} \frac{1}{n} \sup _{\Lambda_{E_{0}^{n}}^{s}, p_{X \mid S}, \varphi_{A}^{x}: I\left(C^{n} S ; X B^{n}\right)_{\rho} \leq L}\left[I\left(X ; B^{n}\right)_{\rho}-I(X ; S)\right] .
$$

2) For a measurement channel $\mathcal{M}_{E A \rightarrow B}$ with a classical CSI system $E_{0} \equiv S$,

$$
\mathbb{C}_{\mathrm{Cl}}(\mathcal{M}, L)=\sup _{p_{X \mid S}, \varphi_{A}^{x}: I(C S ; X Y)_{\rho} \leq L}[I(X ; Y)-I(X ; S)] .
$$

To illustrate our results, we return to the channels in Examples 1 and 2. Example 1 is a trivial example where there is no tradeoff between the transmission rate and the leakage. Specifically, Alice can transmit 1 bit of information per transmission without leakage. Hence, the capacity-leakage region of the random-parameter depolarizing channel is given by

$$
\mathbb{C}_{\mathrm{Cl}}(\mathcal{N})=\left\{(R, L): \begin{array}{l}
R \leq 1 \\
L \geq 0
\end{array}\right\}
$$

Now, we demonstrate the tradeoff for the channel in Example 2.

Example 3. Consider a qubit channel $\mathcal{N}_{S A \rightarrow B}$ that depends on a classical random parameter $S \sim \operatorname{Bernoulli}(\varepsilon)$, hence $E_{0} \equiv E \equiv C \equiv S$. As pointed out in II B, such a random-parameter quantum channel can be viewed as a random selection from a set of channels, $\left\{\mathcal{N}^{(s)}\right\}_{s=0,1}$. Let

$$
\begin{aligned}
& \mathcal{N}^{(0)}(\rho)=\rho \\
& \mathcal{N}^{(1)}(\rho)=|\psi\rangle\langle\psi|
\end{aligned}
$$

where $|\psi\rangle$ is a given state in the same qubit space, as in Example 2. Here, the parameter $S_{i}$ chooses whether the $i$ th input system is projected onto $|\psi\rangle$. This channel has also been considered in the dual model of parameter estimation [32, Example 4]. Ignoring the CSI at the encoder, the average channel $\overline{\mathcal{N}}_{A \rightarrow B}(\rho)=(1-\varepsilon) \rho+\varepsilon|\psi\rangle\langle\psi|$ resembles the quantum erasure channel [68] (see also [66, Section 20.4.3]), except that the "erasure state" of an erasure channel is orthogonal to the qubit space, while $|\psi\rangle$ in the present example is in the same qubit space. Nonetheless, we note that if the decoder knows the locations where the state is projected, then this model is equivalent to the quantum erasure channel. Without this knowledge at the decoder, it is less obvious. 
By Theorem 3, the following rate-leakage region is achievable for the random-parameter channel above,

$$
\mathbb{C}_{\mathrm{Cl}}(\mathcal{N}) \supseteq \bigcup_{0 \leq \alpha \leq \frac{1}{2}}\left\{\begin{array}{ll}
(R, L): & R \leq(1-\varepsilon) h(\alpha) \\
& L \geq h((1-\varepsilon) \alpha)-(1-\varepsilon) h(\alpha)
\end{array}\right\}
$$

where $h(x)$ is the binary entropy function, i.e. $h(x)=-(1-x) \log (1-x)-x \log (x)$ for $x \in(0,1)$, and $h(0)=h(1)=0$. We can see the tradeoff between the communication rate and the leakage. Clearly, if the encoder constantly transmits $|\psi\rangle$, then there is no leakage, as the output is $|\psi\rangle \otimes \cdots \otimes|\psi\rangle$. Yet, the rate is zero as well. Indeed, for $\alpha=0$, we achieve $(R, L)=(0,0)$. On the other hand, taking $\alpha=\frac{1}{2}$, we obtain the maximal rate $R=1-\varepsilon$, which is also the capacity of the quantum erasure channel. However, the leakage is $L=h\left(\frac{1}{2}(1-\varepsilon)\right)-(1-\varepsilon)$.

To show this, note that the bound on the rate on the RHS of (21) can also be expressed as

$$
\begin{aligned}
R & \leq H(X \mid S)-H(X \mid B)_{\rho} \\
& =H(X \mid S)-H(X B)_{\rho}+H(B)_{\rho} .
\end{aligned}
$$

Given CSI at the encoder, we can choose an auxiliary $X$ that depends on the channel parameter $S$. Let the input ensemble be the basis $\left\{|\psi\rangle,\left|\psi_{\perp}\right\rangle\right\}$, where $\left|\psi_{\perp}\right\rangle$ is orthogonal with respect to $|\psi\rangle$. The input distribution is chosen as follows. Let $V \sim \operatorname{Bernoulli}(\alpha)$ be statistically independent of $S$. If $S=0$, set $X=V$. Otherwise, if $S=1$, set $X=0$. This results in the following quantum state,

$$
\begin{aligned}
\rho_{S X B} & =(1-\varepsilon)|0\rangle\langle 0| \otimes\left((1-\alpha)|0\rangle\langle 0|\otimes| \psi\rangle\langle\psi|+\alpha| 1\rangle\left\langle 1|\otimes| \psi_{\perp}\right\rangle\left\langle\psi_{\perp}\right|\right) \\
& +\varepsilon|1\rangle\langle 1|\otimes| 0\rangle\langle 0|\otimes| \psi\rangle\langle\psi| \\
\rho_{X B} & =[(1-\varepsilon)(1-\alpha)+\varepsilon]|0\rangle\langle 0|\otimes| \psi\rangle\langle\psi|+(1-\varepsilon) \alpha| 1\rangle\left\langle 1|\otimes| \psi_{\perp}\right\rangle\left\langle\psi_{\perp}\right|
\end{aligned}
$$

Hence,

$$
\begin{aligned}
H(X B)_{\rho} & =H(B)_{\rho}=h((1-\varepsilon) \alpha) \\
H(X B \mid S)_{\rho} & =H(X \mid S)=(1-\varepsilon) \cdot H(V)+\varepsilon \cdot 0=(1-\varepsilon) h(\alpha)
\end{aligned}
$$

and

$$
I(S ; X B)_{\rho}=H(X B)_{\rho}-H(X B \mid S)_{\rho}=h((1-\varepsilon) \alpha)-(1-\varepsilon) h(\alpha)
$$

\section{SUMMARY AND CONCLUDING REMARKS}

We consider communication of classical information over a quantum state-dependent channel $\mathcal{N}_{E A \rightarrow B}$, when the encoder can measure channel side information (CSI) and is required to mask information on the quantum channel state from the decoder. Specifically, the channel state systems are in an entangled state $\left|\phi_{E_{0} E C}\right\rangle^{\otimes n}$ (see Figure 1). Alice wishes to send a classical message $m$. To this end, she measures the CSI systems $E_{0}^{n}$ and obtains an outcome $V$. Based on the measurement outcome, Alice encodes the quantum state of the channel input systems $A^{n}$ in such a manner that limits the leakage-rate of Bob's information on $C^{n}$ from $B^{n}$.

In quantum channel state masking, analogously to the classical model [6], the channel state system $C^{n}$ store undesired quantum information which leaks to the receiver. This can model a leakage of secret network information in the system to the end-user. Alternatively, the state system $C^{n}$ may represent another transmission to another receiver, Charlie, which is not intended to Bob, and is therefore to be concealed from him. Thus, Alice's goal is to mask this undesired information as much as possible on the one hand, and to transmit reliable information on the other.

In a recent paper by the authors [63], we have considered a quantum state-dependent channel $\mathcal{N}_{E A \rightarrow B}$, when the encoder has CSI and is required to mask information on the quantum channel state from the decoder. We have established a full characterization for the entanglement-assisted masking region with maximally correlated channel state systems, and a regularized formula for the quantum masking region without assistance. Here, we have removed the entanglement assistance, and considered the transmission of classical information over the quantum channel.

Masking can also be viewed as a building block for cryptographic problems of oblivious transfer of information, such as bit commitment or secure computation. Suppose that Alice is a server that receives a query. She is required to use a quantum computer in order to compute a difficult task, while also using a private source $E_{0}^{n} C^{n}$. To this end, Alice uses $E_{0}^{n}$ to encode $A^{n}$, including a reference number $m$ (metadata), which could possibly include the computation query as well. Next, she performs the computation map $\mathcal{N}_{E A \rightarrow B}^{\otimes n}$ on the systems $E^{n} A^{n}$, which are entangled with the private source. The quantum output system $B^{n}$ is delivered to the agent Bob, who performs a measurement to view the metadata $m$, and then use $B^{n}$ as he wishes. The masking requirement is to prevent Bob from recovering the server's private source. 


\section{ACKNOWLEDGMENT}

Uzi Pereg, Christian Deppe, and Holger Boche were supported by the Bundesministerium für Bildung und Forschung (BMBF) through Grants 16KISQ028 (Pereg, Deppe) and 16KIS0858 (Boche). This work of H. Boche was supported in part by the German Federal Ministry of Education and Research (BMBF) within the national initiative for "Post Shannon Communication (NewCom)" under Grant 16KIS1003K and in part by the German Research Foundation (DFG) within the Gottfried Wilhelm Leibniz Prize under Grant BO 1734/20-1 and within Germany's Excellence Strategy EXC-2092 - 390781972 and EXC-2111 - 390814868. U. Pereg was also supported by the Israel CHE Fellowship for Quantum Science and Technology.

\section{Appendix A: Cardinality Bound}

Consider the region $\mathcal{R}_{\mathrm{Cl}}(\mathcal{N})$ as defined in $(21)$. To bound the alphabet size, we use the Fenchel-EgglestonCarathéodory lemma [69] and similar arguments as in in [32]. First, observe that since $\Lambda_{E_{0}}^{s}$ is a measurement on $E_{0}$, we can restrict the dimension of this measurement to $\left|\mathcal{H}_{E_{0}}\right|$, hence $|\mathcal{S}| \leq\left|\mathcal{H}_{E_{0}}\right|$. Fix $p_{S}(s)=\operatorname{Tr}\left(\Lambda_{E_{0}}^{s} \phi_{E_{0}}\right)$, and consider the ensemble $\left\{p_{X \mid S}(x \mid s), \varphi_{A}^{x}\right\}$. Every quantum state $\theta_{A}$ has a unique parametric representation $u\left(\theta_{A}\right)$ of dimension $\left|\mathcal{H}_{A}\right|^{2}-1$ (see [32, Appendix B]). Then, define a map $f_{s}: \mathcal{X} \rightarrow \mathbb{R}^{\left|\mathcal{H}_{A}\right|^{2}+1}$ by

$$
f_{s}(x)=\left(u\left(\varphi_{A}^{x}\right),-H(B \mid X=x)_{\rho}+H(S \mid X=x), H(C S \mid B, X=x)_{\rho}\right) .
$$

The map $f_{s}$ can be extended to probability distributions as follows,

$$
F_{s}: p_{X \mid S}(\cdot \mid s) \mapsto \sum_{x \in \mathcal{X}} p_{X \mid S}(x \mid s) f_{s}(x)=\left(u\left(\rho_{A}^{s}\right),-H(B \mid X)_{\rho}+H(S \mid X), H(C S \mid B X)_{\rho}\right)
$$

for $s \in \mathcal{S}$, where $\rho_{A}^{s}=\sum_{x} p_{X \mid S}(x \mid s) \varphi_{A}^{x}$. According to the Fenchel-Eggleston-Carathéodory lemma [69], any point in the convex closure of a connected compact set within $\mathbb{R}^{d}$ belongs to the convex hull of $d$ points in the set. Since the map $F_{s}$ is linear, it maps the set of distributions on $\mathcal{X}$ to a connected compact set in $\mathbb{R}^{\left|\mathcal{H}_{A}\right|^{2}+1}$. Thus, for every $s$, there exists a conditional probability distribution $p_{\bar{X} \mid S}(\cdot \mid s)$ on a subset $\overline{\mathcal{X}} \subseteq \mathcal{X}$ of size $\left|\mathcal{H}_{A}\right|^{2}+1$, such that $F_{S}\left(p_{\bar{X} \mid S}(\cdot \mid s)\right)=F_{S}\left(p_{X \mid S}(\cdot \mid s)\right)$. We deduce that the alphabet dimension can be restricted to $|\mathcal{X}| \leq\left(\left|\mathcal{H}_{A}\right|^{2}+1\right)\left|\mathcal{H}_{E_{0}}\right|$, while preserving $\rho_{S A E C}$ and $\rho_{S B C} \equiv \mathcal{N}_{E A \rightarrow B}\left(\rho_{S E A C}\right) ; I(X ; B)_{\rho}-I(X ; S)=H(B)_{\rho}-H(B \mid X)_{\rho}+H(S \mid X)-H(S)$; and $I(C S ; X B)_{\rho}=H(C S)_{\rho}-H(C S \mid B X)_{\rho}$.

\section{Appendix B: Information Theoretic Tools}

To derive our results, we use the quantum version of the method of types properties and techniques. The basic definitions and lemmas are similar to those in [32]. For convenience, we bring them here as well.

\section{Classical Types}

The type of a classical sequence $x^{n}$ is defined as the empirical distribution $\hat{P}_{x^{n}}(a)=N\left(a \mid x^{n}\right) / n$ for $a \in \mathcal{X}$, where $N\left(a \mid x^{n}\right)$ is the number of occurrences of the symbol $a$ in the sequence $x^{n}$. Denote the set of all types over $\mathcal{X}$ is by $\mathcal{P}_{n}(\mathcal{X})$. For a pair of sequences $x^{n}$ and $y^{n}$, we give similar definitions in terms of the joint type $\hat{P}_{x^{n}, y^{n}}(a, b)=N\left(a, b \mid x^{n}, y^{n}\right) / n$ for $a \in \mathcal{X}, b \in \mathcal{Y}$, where $N\left(a, b \mid x^{n}, y^{n}\right)$ is the number of occurrences of the symbol pair $(a, b)$ in the sequence $\left(x_{i}, y_{i}\right)_{i=1}^{n}$. Given a sequence $y^{n} \in \mathcal{Y}^{n}$, we further define the conditional type $\hat{P}_{x^{n} \mid y^{n}}(a \mid b)=N\left(a, b \mid x^{n}, y^{n}\right) / N\left(b \mid y^{n}\right)$.

Given a probability distribution $p_{X} \in \mathcal{P}(\mathcal{X})$, define the $\delta$-typical set as

$$
\begin{aligned}
\mathcal{A}^{\delta}\left(p_{X}\right) \equiv\left\{x^{n} \in \mathcal{X}^{n}:\left|\hat{P}_{x^{n}}(a)-p_{X}(a)\right| \leq \delta\right. & \text { if } p_{X}(a)>0 \\
\hat{P}_{x^{n}}(a)=0 & \text { if } \left.p_{X}(a)=0, \forall a \in \mathcal{X}\right\}
\end{aligned}
$$

The covering lemma is a powerful tool in classical information theory [70]. 
Lemma 5 (Classical Covering Lemma [70][71, Lemma 3.3]). Let $X^{n} \sim \prod_{i=1}^{n} p_{X}\left(x_{i}\right), \delta>0$, and let $Z^{n}(m), m \in$ $\left[1: 2^{n R}\right]$, be independent random sequences distributed according to $\prod_{i=1}^{n} p_{Z}\left(z_{i}\right)$. Suppose that the sequence $X^{n}$ is pairwise independent of the sequences $Z^{n}(m), m \in\left[1: 2^{n R}\right]$. Then,

$$
\operatorname{Pr}\left(\left(Z^{n}(m), X^{n}\right) \notin \mathcal{A}^{\delta}\left(p_{Z, X}\right) \text { for all } m \in\left[1: 2^{n R}\right]\right) \leq \exp \left(-2^{n\left(R-I(Z ; X)-\varepsilon_{n}(\delta)\right.}\right)
$$

where $\varepsilon_{n}(\delta)$ tends to zero as $n \rightarrow \infty$ and $\delta \rightarrow 0$.

Let $X^{n} \sim \prod_{i=1}^{n} p_{X}\left(x_{i}\right)$ be an information source sequence, encoded by an index $m$ at compression rate $R$. Based on the covering lemma above, as long as the compression rate is higher than $I(Z ; X)$, a set of random codewords, $Z^{n}(m) \sim \prod_{i=1}^{n} p_{Z}\left(z_{i}\right)$, contains with high probability at least one sequence that is jointly typical with the source sequence.

Though originally stated in the context of lossy source coding, the classical covering lemma is useful in a variety of scenarios [71], including communication with CSI [32]. In our analysis in the sequel, we will have a measurement sequence $S^{n}$ playing the role of the "source sequence".

\section{Quantum Typical Subspaces}

Moving to the quantum method of types, suppose that the state of a system is generated from an ensemble $\left\{p_{X}(x),|x\rangle\right\}_{x \in \mathcal{X}}$, hence, the average density operator is

$$
\rho=\sum_{x \in \mathcal{X}} p_{X}(x)|x\rangle\langle x| .
$$

Consider the subspace spanned by the vectors $\left|x^{n}\right\rangle$, for $x^{n} \in \mathcal{A}^{\delta}\left(p_{X}\right)$. The projector onto the subspace is defined as

$$
\Pi^{\delta}(\rho) \equiv \sum_{x^{n} \in \mathcal{A}^{\delta}\left(p_{X}\right)}\left|x^{n}\right\rangle\left\langle x^{n}\right| .
$$

Based on [72] [73, Theorem 12.5], for every $\varepsilon, \delta>0$ and sufficiently large $n$, the $\delta$-typical projector satisfies

$$
\begin{aligned}
\operatorname{Tr}\left(\Pi^{\delta}(\rho) \rho^{\otimes n}\right) & \geq 1-\varepsilon \\
2^{-n(H(\rho)+c \delta)} \Pi^{\delta}(\rho) & \preceq \Pi^{\delta}(\rho) \rho^{\otimes n} \Pi^{\delta}(\rho) \preceq 2^{-n(H(\rho)-c \delta)} \\
\operatorname{Tr}\left(\Pi^{\delta}(\rho)\right) & \leq 2^{n(H(\rho)+c \delta)}
\end{aligned}
$$

where $c>0$ is a constant.

We will also need the conditional $\delta$-typical subspace. Consider a state

$$
\sigma=\sum_{x \in \mathcal{Y}} p_{X}(x) \rho_{B}^{x}
$$

with

$$
\rho_{B}^{x}=\sum_{y \in \mathcal{Y}} p_{Y \mid X}(y \mid x)\left|\psi^{x, y}\right\rangle\left\langle\psi^{x, y}\right| .
$$

Given a fixed sequence $x^{n} \in \mathcal{X}^{n}$, divide the index set $[1: n]$ into the subsets $I_{n}(a)=\left\{i: x_{i}=a\right\}, a \in \mathcal{X}$, and define the conditional $\delta$-typical subspace $\mathscr{S}^{\delta}\left(\sigma_{B} \mid x^{n}\right)$ as the span of the vectors $\left|\psi^{x^{n}, y^{n}}\right\rangle=\otimes_{i=1}^{n}\left|\psi^{x_{i}, y_{i}}\right\rangle$ such that

$$
y^{I_{n}(a)} \in \mathcal{A}_{\delta}^{\left(\left|I_{n}(a)\right|\right)}\left(p_{Y \mid X=a}\right), \text { for } a \in \mathcal{X} .
$$

The projector onto the conditional $\delta$-typical subspace is defined as

$$
\Pi^{\delta}\left(\sigma_{B} \mid x^{n}\right) \equiv \sum_{\left|\psi^{x^{n}, y^{n}}\right\rangle \in \mathscr{S}^{\delta}\left(\sigma_{B} \mid x^{n}\right)}\left|\psi^{x^{n}, y^{n}}\right\rangle\left\langle\psi^{x^{n}, y^{n}}\right| .
$$

Based on [72] [66, Section 15.2.4], for every $\varepsilon^{\prime}, \delta>0$ and sufficiently large $n$,

$$
\begin{aligned}
\operatorname{Tr}\left(\Pi^{\delta}\left(\sigma_{B} \mid x^{n}\right) \rho_{B^{n}}^{x^{n}}\right) & \geq 1-\varepsilon^{\prime} \\
2^{-n\left(H\left(B \mid X^{\prime}\right)_{\sigma}+c^{\prime} \delta\right)} \Pi^{\delta}\left(\sigma_{B} \mid x^{n}\right) & \preceq \Pi^{\delta}\left(\sigma_{B} \mid x^{n}\right) \rho_{B^{n}}^{x^{n}} \Pi^{\delta}\left(\sigma_{B} \mid x^{n}\right) \preceq 2^{-n\left(H\left(B \mid X^{\prime}\right)_{\sigma}-c^{\prime} \delta\right)} \\
\operatorname{Tr}\left(\Pi^{\delta}\left(\sigma_{B} \mid x^{n}\right)\right) & \leq 2^{n\left(H\left(B \mid X^{\prime}\right)_{\sigma}+c^{\prime} \delta\right)}
\end{aligned}
$$


where $c^{\prime}>0$ is a constant, $\rho_{B^{n}}^{x^{n}}=\bigotimes_{i=1}^{n} \rho_{B_{i}}^{x_{i}}$, and the classical random variable $X^{\prime}$ is distributed according to the type of $x^{n}$. Furthermore, if $x^{n} \in \mathcal{A}^{\delta}\left(p_{X}\right)$, then

$$
\operatorname{Tr}\left(\Pi^{\delta}\left(\sigma_{B}\right) \rho_{B^{n}}^{x^{n}}\right) \geq 1-\varepsilon^{\prime}
$$

(see [66, Property 15.2.7]).

\section{Quantum Packing Lemma}

To prove achievability for the HSW Theorem (see Theorem 1), one may invoke the quantum packing lemma [65, 66]. Suppose that Alice employs a codebook that consists of $2^{n R}$ codewords $x^{n}(m), m \in\left[1: 2^{n R}\right]$, by which she chooses a quantum state from an ensemble $\left\{\rho_{x^{n}}\right\}_{x^{n} \in \mathcal{X}^{n}}$. The proof is based on random codebook generation, where the codewords are drawn at random according to an input distribution $p_{X}(x)$. To recover the transmitted message, Bob may perform the square-root measurement $[23,24]$ using a code projector $\Pi$ and codeword projectors $\Pi_{x^{n}}, x^{n} \in \mathcal{X}^{n}$, which project onto subspaces of the Hilbert space $\mathcal{H}_{B^{n}}$.

The lemma below is a simplified, less general, version of the quantum packing lemma by Hsieh, Devetak, and Winter [65].

Lemma 6 (Quantum Packing Lemma [65, Lemma 2]). Let

$$
\rho=\sum_{x \in \mathcal{X}} p_{X}(x) \rho_{x}
$$

where $\left\{p_{X}(x), \rho_{x}\right\}_{x \in \mathcal{X}}$ is a given ensemble. Furthermore, suppose that there is a code projector $\Pi$ and codeword projectors $\Pi_{x^{n}}, x^{n} \in \mathcal{A}^{\delta}\left(p_{X}\right)$, that satisfy for every $\alpha>0$ and sufficiently large $n$,

$$
\begin{aligned}
& \operatorname{Tr}\left(\Pi \rho_{x^{n}}\right) \geq 1-\alpha \\
& \operatorname{Tr}\left(\Pi_{x^{n}} \rho_{x^{n}}\right) \geq 1-\alpha \\
& \operatorname{Tr}\left(\Pi_{x^{n}}\right) \leq 2^{n d} \\
& \Pi \rho^{\otimes n} \Pi \preceq 2^{-n(D-\alpha)} \Pi
\end{aligned}
$$

for some $0<d<D$ with $\rho_{x^{n}} \equiv \bigotimes_{i=1}^{n} \rho_{x_{i}}$. Then, there exist codewords $x^{n}(m), m \in\left[1: 2^{n R}\right]$, and a POVM $\left\{\Lambda_{m}\right\}_{m \in\left[1: 2^{n R}\right]}$ such that

$$
\operatorname{Tr}\left(\Lambda_{m} \rho_{x^{n}(m)}\right) \geq 1-2^{-n\left[D-d-R-\varepsilon_{n}(\alpha)\right]}
$$

for all $m \in\left[1: 2^{n R}\right]$, where $\varepsilon_{n}(\alpha)$ tends to zero as $n \rightarrow \infty$ and $\alpha \rightarrow 0$.

In our analysis, where there is CSI at the encoder, we apply the packing lemma such that the quantum ensemble encodes both the message $m$ and a compressed representation of the parameter sequence $s^{n}$.

\section{Appendix C: Proof of Theorem 3}

Consider a quantum state-dependent channel $\mathcal{N}_{E A \rightarrow B}$ with CSI at the encoder.

\section{Part 1}

\section{Direct Part}

We show that for every $\zeta_{0}, \varepsilon_{0}, \delta_{0}>0$, there exists a $\left(2^{n\left(R-\zeta_{0}\right)}, n, \varepsilon_{0}, L+\delta_{0}\right)$ code for $\mathcal{N}_{E A \rightarrow B}$, provided that $(R, L) \in \mathcal{R}_{\mathrm{Cl}}(\mathcal{N})$. To prove achievability, we extend the classical binning technique and apply the quantum packing lemma and classical covering lemma.

The code construction, encoding and decoding procedures are described below. 
a. Classical Codebook Construction

Let $\delta>0$, and let $\widetilde{R}>R$ be chosen later. We construct $2^{n R}$ sub-codebooks at random. For every message $m \in\left[1: 2^{n R}\right]$, select $2^{n(\widetilde{R}-R)}$ independent sequences $x^{n}(k)$ at random, each according to $\prod_{i=1}^{n} p_{X}\left(x_{i}\right)$. Then, we have the following sub-codebooks,

$$
\mathscr{B}(m)=\left\{x^{n}(k): k \in\left[(m-1) 2^{n(\widetilde{R}-R)}+1: m 2^{n(\widetilde{R}-R)}\right]\right\}
$$

for $m \in\left[1: 2^{n R}\right]$.

\section{b. Encoding and Decoding}

To send a message $m$, Alice performs the following.

(i) Measure the CSI systems $E_{0, i}$ using the POVM $\Lambda_{E_{0}}^{s}$, for $i \in[1: n]$. Since the CSI systems are in a product state, the measurement outcome is an i.i.d. sequence $\sim q(s)$, where $q(s)=\operatorname{Tr}\left(\Lambda_{E_{0}}^{s} \sigma_{E_{0}}\right)$.

(ii) Given a measurement outcome $s^{n}$, find a sequence $x^{n}(k) \in \mathscr{B}(m)$ such that $\left(s^{n}, x^{n}(k)\right) \in \mathcal{A}^{\delta}\left(p_{S, X}\right)$, where $p_{S, X}(s, u)=q(s) p_{X \mid S}(u \mid s)$. If there is none, select $x^{n}(k)$ arbitrarily, and if there is more than one such sequence, choose the first among them.

(iii) Transmit $\rho_{A^{n}}^{m}=\bigotimes_{i=1}^{n} \varphi_{A}^{x_{i}(k)}$.

Bob receives the output system $B^{n}$, such that

$$
\rho_{B^{n}}^{m}=\bigotimes_{i=1}^{n} \rho_{B}^{x_{i}(k)}
$$

and decodes $\hat{k}$ by applying a POVM $\left\{\Lambda_{k}\right\}_{k \in\left[1: 2^{n \tilde{R}}\right]}$, which will be specified later. He declares his estimate $\hat{m}$ to be the corresponding sub-codebook index, i.e. $\hat{m}$ such that $x^{n}(\hat{k}) \in \mathscr{B}(\hat{m})$.

\section{Analysis of Probability of Error and Leakage}

First, we show that the probability of decoding error tends to zero as $n \rightarrow \infty$. By symmetry, we may assume without loss of generality that Alice sends the message $M=1$ using $K$. Consider the following events,

$$
\begin{aligned}
& \mathscr{E}_{1}=\left\{\left(S^{n}, X^{n}\left(k^{\prime}\right)\right) \notin \mathcal{A}^{\delta}\left(p_{S, X}\right), \text { for all } k^{\prime} \in \mathscr{B}(1)\right\} \\
& \mathscr{E}_{2}=\{\hat{K} \neq K\}
\end{aligned}
$$

By the union of events bound, the probability of error is bounded by

$$
P_{e}^{(n)}(\mathcal{T}, \mathcal{F}, \mathcal{D}) \leq \operatorname{Pr}\left(\mathscr{E}_{1}\right)+\operatorname{Pr}\left(\mathscr{E}_{2} \mid \mathscr{E}_{1}^{c}\right)
$$

where the conditioning on $M=1$ is omitted for convenience of notation. By the classical covering lemma, Lemma 5 , the first term tends to zero as $n \rightarrow \infty$ for

$$
\widetilde{R}-R>I(X ; S)+\varepsilon_{1}(\delta) .
$$

Hence, we choose

$$
\widetilde{R}=R+I(X ; S)+2 \varepsilon_{1}(\delta) .
$$

To bound the second term, we use the quantum packing lemma. Given $\mathscr{E}_{1}^{c}$, we have $X^{n}(K) \in \mathcal{A}^{\delta_{1}}\left(p_{X}\right)$, with $\delta_{1} \triangleq \delta|\mathcal{S}|$. Next, observe that

$$
\begin{aligned}
\Pi^{\delta}\left(\rho_{B}\right) \rho_{B^{n}} \Pi^{\delta}\left(\rho_{B}\right) & \preceq 2^{-n\left(H(B)_{\rho}-\varepsilon_{2}(\delta)\right)} \Pi^{\delta}\left(\rho_{B}\right) \\
\operatorname{Tr}\left[\Pi^{\delta}\left(\rho_{B} \mid x^{n}\right) \rho_{B^{n}}^{x^{n}}\right] & \geq 1-\varepsilon_{2}(\delta) \\
\operatorname{Tr}\left[\Pi^{\delta}\left(\rho_{B} \mid x^{n}\right)\right] & \leq 2^{n\left(H(B \mid X)_{\rho}+\varepsilon_{2}(\delta)\right)} \\
\operatorname{Tr}\left[\Pi^{\delta}\left(\rho_{B}\right) \rho_{B^{n}}^{x^{n}}\right] & \geq 1-\varepsilon_{2}(\delta)
\end{aligned}
$$


for $x^{n} \in \mathcal{A}^{\delta_{1}}\left(p_{X}\right)$, by (B6), (B12), (B14), and (B15), respectively. Thus, by Lemma 6 , there exists a POVM $D_{k}$ such that the second error term in $(\mathrm{C} 5)$ is bounded by $\operatorname{Pr}\left(\mathscr{E}_{2} \mid \mathscr{E}_{1}^{c}\right) \leq 2^{-n\left(I(X ; B)_{\rho}-\widetilde{R}-\varepsilon_{3}(\delta)\right)}$, which tends to zero as $n \rightarrow \infty$, if

$$
\widetilde{R}<I(X ; B)_{\rho}-\varepsilon_{3}(\delta) .
$$

Hence, by (C7), the probability of decoding error tends to zero, provided that the transmission rate is bounded by

$$
R<I(X ; B)_{\rho}-I(X ; S)-\varepsilon_{3}(\delta)-2 \varepsilon_{2}(\delta) .
$$

As for the leakage rate, observe that

$$
\begin{aligned}
I\left(C^{n} ; B^{n}\right)_{\rho} & \leq I\left(C^{n} ; X^{n}(K), B^{n}\right)_{\rho} \\
& =I\left(C^{n} ; X^{n}(K)\right)_{\rho}+I\left(C^{n} ; B^{n} \mid X^{n}(K)\right)_{\rho} .
\end{aligned}
$$

Then, the first term is bounded by

$$
\begin{aligned}
I\left(C^{n} ; X^{n}(K)\right)_{\rho} & \leq I\left(C^{n} ; M, X^{n}(K)\right)_{\rho} \\
& \stackrel{(a)}{=} I\left(C^{n} ; X^{n}(K) \mid M\right)_{\rho} \\
& \leq H\left(X^{n}(K) \mid M\right)_{\rho} \\
& \stackrel{(b)}{\leq} n(\widetilde{R}-R) \\
& \stackrel{(c)}{=} n\left(I(X ; S)+2 \varepsilon_{1}(\delta)\right) \\
& \leq n\left(I(X ; C, S)+2 \varepsilon_{1}(\delta)\right)
\end{aligned}
$$

where $(a)$ holds since $I\left(C^{n} ; M\right)_{\rho}=0$, as there is no correlation between the classical message $M$ and the channel state system $C^{n},(b)$ follows as $X^{n}(K)$ belongs to a sub-codebook $\mathscr{B}(M)$ of size $2^{n(\widetilde{R}-R)}$, and $(c)$ is due to (C7). Moving to the second term in the RHS of (C14),

$$
\begin{aligned}
I\left(C^{n} ; B^{n} \mid X^{n}(K)\right)_{\rho} & \leq I\left(C^{n}, S^{n} ; B^{n} \mid X^{n}(K)\right)_{\rho} \\
& =H\left(B^{n} \mid X^{n}(K)\right)_{\rho}-H\left(B^{n} \mid C^{n}, S^{n}, X^{n}(K)\right)_{\rho}
\end{aligned}
$$

Now, since conditioning does not increase the quantum entropy,

$$
H\left(B^{n} \mid X^{n}(K)\right)_{\rho} \leq \sum_{i=1}^{n} H\left(B_{i} \mid X_{i}(K)\right)_{\rho}=n H(B \mid X)_{\rho} .
$$

Furthermore, given $X^{n}(K)=x^{n}$ and $S^{n}=s^{n}$, we have a product output state $\rho_{B^{n} C^{n}} \equiv \bigotimes_{i=1}^{n} \mathcal{N}_{E A \rightarrow B}\left(\sigma_{E C}^{s_{i}} \otimes \varphi_{A}^{x_{i}, s_{i}}\right)$, where $\sigma_{E C}^{s}$ denotes the post-measurement state, i.e. $\sigma_{E C}^{s} \equiv \operatorname{Tr}_{E_{0}}\left(\Lambda_{E_{0}}^{s} \phi_{E_{0} E C}\right) / \operatorname{Tr}\left(\Lambda_{E_{0}}^{s} \phi_{E_{0}}\right)$ for $s \in \mathcal{S}$. Thus,

$$
H\left(B^{n} \mid C^{n}, S^{n}, X^{n}(K)\right)_{\rho}=n H(B \mid C, S, X)_{\rho} .
$$

It follows from (C14)-(C18) that

$$
\begin{aligned}
\frac{1}{n} I\left(B^{n} ; C^{n}\right) & \leq I(X ; C, S)+2 \varepsilon_{1}(\delta)+H(B \mid X)_{\rho}-H(B \mid C, S, X)_{\rho} \\
& =I(C, S ; X)+I(C, S ; B \mid X)+2 \varepsilon_{1}(\delta) \\
& =I(C, S ; X, B)+2 \varepsilon_{1}(\delta) .
\end{aligned}
$$

Thereby, the leakage requirement holds if

$$
I(C, S ; X, B) \leq L-2 \varepsilon_{1}(\delta) .
$$

To show that rate-leakage pairs in the regularized formula, $\frac{1}{\kappa} \mathcal{R}_{\mathrm{Cl}}\left(\mathcal{N}^{\otimes \kappa}\right)$, are achievable as well, one may use the coding scheme above over the product channel $\mathcal{N}^{\otimes \kappa}$, where $\kappa$ is arbitrarily large. This completes the proof of the direct part. 


\section{Converse Part}

The proof of the regularized converse part is a straightforward extension of standard considerations. For completeness, we give the details below. Suppose that Alice and Bob are trying to distribute randomness. An upper bound on the rate at which Alice can distribute randomness to Bob also serves as an upper bound on the classical communication rate. Then, suppose that Alice prepares a maximally correlated state

$$
\pi_{M M^{\prime}} \equiv \frac{1}{2^{n R}} \sum_{m=1}^{2^{n R}}|m\rangle\left\langle\left. m\right|_{M} \otimes \mid m\right\rangle\left\langle\left. m\right|_{M^{\prime}}\right.
$$

locally, where $M$ and $M^{\prime}$ are classical message registers. Denote the joint state at the beginning by

$$
\psi_{M M^{\prime} E_{0}^{n} E^{n} C^{n}}=\pi_{M M^{\prime}} \otimes \phi_{E_{0} E C}^{\otimes n}
$$

where $E^{n}$ are the channel state systems, $E_{0}^{n}$ are the CSI systems that are available to Alice, and $C^{n}$ are the systems that are masked from Bob (see Figure 1).

Alice performs a measurement $\mathcal{T}_{E_{0}^{n} \rightarrow V}$ on the CSI systems $E_{0}^{n}$, and obtains a measurement outcome $V$. Denote the average post-measurement state by

$$
\rho_{M M^{\prime} V E^{n} C^{n}} \equiv \mathcal{T}_{E_{0}^{n} \rightarrow V}\left(\psi_{M M^{\prime} E_{0}^{n} E^{n} C^{n}}\right)
$$

Then, she applies an encoding map $\mathcal{F}_{M^{\prime} V \rightarrow A^{n} V}$ to the classical system $M^{\prime}$ and the measurement outcome $V$ (since $V$ is classical, it can be copied.) The resulting state is

$$
\rho_{M A^{n} V E^{n} C^{n}} \equiv \mathcal{F}_{M^{\prime} V \rightarrow A^{n} V}\left(\rho_{M M^{\prime} V E^{n} C^{n}}\right) .
$$

As the input systems $A^{n}$ are sent through the channel, the output state is

$$
\rho_{M B^{n} C^{n} V} \equiv \mathcal{N}_{E A \rightarrow B}^{\otimes n}\left(\rho_{\left.M E^{n} A^{n} C^{n} V\right)}\right.
$$

Bob receives $B^{n}$ and performs a decoding channel $\mathcal{D}_{B^{n} \rightarrow \hat{M}}$, producing

$$
\rho_{M \hat{M} C^{n} V} \equiv \mathcal{D}_{B^{n} \rightarrow \hat{M}}\left(\rho_{M B^{n} C^{n} V}\right) .
$$

Consider a sequence of codes $\left(\mathcal{T}_{n}, \mathcal{F}_{n}, \mathcal{D}_{n}\right)$ such that

$$
\begin{aligned}
\frac{1}{2}\left\|\rho_{M \hat{M}}-\pi_{M M^{\prime}}\right\|_{1} & \leq \varepsilon_{n} \\
\frac{1}{n} I\left(C^{n} V ; B^{n}\right)_{\rho} & \leq L+\delta_{n}
\end{aligned}
$$

where $\varepsilon_{n}, \delta_{n}$ tend to zero as $n \rightarrow \infty$. Based on the Alicki-Fannes-Winter inequality [74] [66, Theorem 11.10.3], (C27) implies

$$
\left|H(M \mid \hat{M})_{\rho}-H\left(M \mid M^{\prime}\right)_{\pi}\right| \leq n \varepsilon_{n}^{\prime}
$$

where $\varepsilon_{n}^{\prime} \rightarrow 0$ as $n \rightarrow \infty$. Since $H\left(\pi_{M M^{\prime}}\right)=H\left(\pi_{M}\right)=H\left(\pi_{M^{\prime}}\right)=n R$, we have $I(M ; \hat{M})_{\pi}=n R$. Then, as $H\left(\rho_{M}\right)=H\left(\pi_{M}\right)=n R$, we also have $I\left(M ; M^{\prime}\right)_{\pi}-I(M ; \hat{M})_{\rho}=H(M \mid \hat{M})_{\rho}-H\left(M \mid M^{\prime}\right)_{\pi}$. Thus, (C29) implies

$$
\begin{aligned}
n R & =I(M ; \hat{M})_{\pi} \\
& \leq I(M ; \hat{M})_{\rho}+n \varepsilon_{n}^{\prime} \\
& \leq I\left(M ; B^{n}\right)_{\rho}+n \varepsilon_{n}^{\prime}
\end{aligned}
$$

where the last line follows from (C26) and the quantum data processing inequality [73, Theorem 11.5]. Since the message has no correlation with the channel state system $E_{0}^{n}$, we can also write this as

$$
\begin{aligned}
n R & \leq I\left(M ; B^{n}\right)_{\rho}-I(M ; V)_{\rho}+n \varepsilon_{n}^{\prime} \\
& =I\left(X^{n} ; B^{n}\right)_{\rho}-I\left(M ; S^{n}\right)_{\rho}+n \varepsilon_{n}^{\prime}
\end{aligned}
$$

as we define $X^{n}=f(M)$ and $S^{n}=g(V)$, where $f$ and $g$ are arbitrary one-to-one maps. This concludes the converse proof for part 1 . 


\section{Part 2}

Now, we consider the special case of a measurement channel $\mathcal{M}_{E A \rightarrow Y}$, where the CSI system and the channel output are classical, i.e. $E_{0} \equiv S \sim q(s)$ and $B \equiv Y$. The direct part follows from part 1. To prove the converse part, we extend the methods of Merhav and Shamai [6].

By the classical chain rule,

$$
\begin{aligned}
I\left(M ; Y^{n}\right) & =\sum_{i=1}^{n} I\left(M ; Y_{i} \mid Y^{i-1}\right) \\
& =\sum_{i=1}^{n} I\left(M Y^{i-1} S_{i+1}^{n} ; Y_{i}\right)-\sum_{i=1}^{n} I\left(Y_{i} ; S_{i+1}^{n} \mid M Y^{i-1}\right) \\
& =\sum_{i=1}^{n} I\left(M Y^{i-1} S_{i+1}^{n} ; Y_{i}\right)-\sum_{i=1}^{n} I\left(Y^{i-1} ; S_{i} \mid M S_{i+1}^{n}\right)
\end{aligned}
$$

where the last line follows from the Csiszár sum identity [71, Section 2.3]. Since $S_{i}$ and $\left(M, S_{i+1}^{n}\right)$ are statistically independent, we have $I\left(Y^{i-1} ; S_{i} \mid M S_{i+1}^{n}\right)=I\left(M S_{i+1}^{n} Y^{i-1} ; S_{i}\right)$. Therefore, defining

$$
X_{i}=\left(M, Y^{i-1}, S_{i+1}^{n}\right)
$$

we obtain

$$
I\left(M ; Y^{n}\right)_{\rho} \leq \sum_{i=1}^{n} I\left(X_{i} ; Y_{i}\right)_{\rho}-\sum_{i=1}^{n} I\left(X_{i} ; S_{i}\right)_{\rho} .
$$

Let $J$ be a classical random variable with a uniform distribution over $\{1, \ldots, n\}$, in a product state with the previous quantum systems, i.e. $C^{n}, E^{n}, E_{0}^{n}, M, M^{\prime}, A^{n}$, and $Y^{n}$. Then, by (C30) and (C34),

$$
\begin{aligned}
R-\varepsilon_{n}^{\prime} & \leq \frac{1}{n} \sum_{i=1}^{n}\left[I\left(X_{i} ; Y_{i}\right)_{\rho}-I\left(X_{i} ; S_{i}\right)_{\rho}\right] \\
& =I\left(X_{J} ; Y_{J} \mid J\right)-I\left(X_{J} ; S_{J} \mid J\right) \\
& =I\left(X_{J}, J ; Y_{J}\right)-I\left(J ; Y_{J}\right)-I\left(X_{J}, J ; S_{J}\right)_{\rho}+I\left(J ; S_{J}\right)_{\rho} \\
& \leq I\left(X_{J}, J ; Y_{J}\right)_{\rho}-I\left(X_{J}, J ; S_{J}\right)_{\rho}+I\left(J ; S_{J}\right)_{\rho} \\
& =I\left(X_{J}, J ; Y_{J}\right)_{\rho}-I\left(X_{J}, J ; S_{J}\right)_{\rho}
\end{aligned}
$$

with $\rho_{J X_{J} E_{J} C_{J} A_{J}}=\frac{1}{n} \sum_{i=1}^{n}|i\rangle\langle i| \otimes \rho_{X_{i} E_{i} C_{i} A_{i}}$ and $\rho_{J X_{J} C_{J} Y_{J}}=\mathcal{M}_{E A \rightarrow Y}\left(\rho_{J X_{J} C_{J} E_{J} A_{J}}\right)$, where the last equality holds since the sequence $S^{n}$ is i.i.d. Thus, defining

$$
X \equiv\left(X_{J}, J\right), S \equiv S_{J}, E \equiv E_{J}, C \equiv C_{J}, A \equiv A_{J}
$$

and $Y$ such that $\rho_{Y C}=\mathcal{M}_{E A \rightarrow Y}\left(\rho_{E A C}\right)$, we obtain the desired bound on the coding rate,

$$
R-\varepsilon_{n}^{\prime} \leq I(X ; Y)-I(X ; S)
$$

As for the leakage rate, by (C28),

$$
\begin{aligned}
n\left(L+\delta_{n}\right) & \geq I\left(C^{n} S^{n} ; Y^{n}\right)_{\rho} \\
& =I\left(C^{n} S^{n} ; Y^{n} M\right)_{\rho}-I\left(C^{n} S^{n} ; M \mid Y^{n}\right)_{\rho} \\
& =I\left(C^{n} S^{n} ; Y^{n} M\right)_{\rho}-H\left(M \mid Y^{n}\right)_{\rho}+H\left(M \mid C^{n} S^{n} Y^{n}\right)_{\rho}
\end{aligned}
$$

For a classical-quantum state $\rho_{X A}=\sum_{x \in \mathcal{X}} p_{X}(x)|x\rangle\langle x| \otimes \rho_{A}^{x}$, the conditional entropy of is always nonnegative, as $H(X \mid A)_{\rho} \geq H(X \mid A, X)=0$, since conditioning cannot increase quantum entropy [73, Theorem 11.15]. As the message $M$ is classical, the last term in the RHS of (C38) is nonnegative, i.e.

$$
H\left(M \mid C^{n}, Y^{n}\right)_{\rho} \geq 0
$$


Furthermore, by (C30), the second term satisfies

$$
H\left(M \mid Y^{n}\right)_{\rho}=H(M)_{\pi}-I\left(M ; Y^{n}\right)_{\rho} \leq n \varepsilon_{n}^{\prime}
$$

Thus, by (C38)-(C40),

$$
\begin{aligned}
n\left(L+\varepsilon_{n}^{\prime}+\delta_{n}\right) & \geq I\left(C^{n} S^{n} ; Y^{n} M\right)_{\rho} \\
& =\sum_{i=1}^{n} I\left(C_{i} S_{i} ; Y^{n} M \mid C_{i+1}^{n} S_{i+1}^{n}\right)_{\rho} \\
& \geq \sum_{i=1}^{n} I\left(C_{i} S_{i} ; Y_{i} Y^{i-1} M \mid C_{i+1}^{n} S_{i+1}^{n}\right)_{\rho} .
\end{aligned}
$$

Then, since $\left(C_{i}, S_{i}\right)$ and $\left(C_{i+1}^{n}, S_{i+1}^{n}\right)$ are in a product state, we have $I\left(C_{i} S_{i} ; C_{i+1}^{n} S_{i+1}^{n}\right)_{\rho}=0$. Hence,

$$
\begin{aligned}
L+\varepsilon_{n}^{\prime}+\delta_{n} & \geq \frac{1}{n} \sum_{i=1}^{n} I\left(C_{i} S_{i} ; Y_{i} Y^{i-1} M C_{i+1}^{n} S_{i+1}^{n}\right)_{\rho} \\
& \geq \frac{1}{n} \sum_{i=1}^{n} I\left(C_{i} S_{i} ; Y_{i} Y^{i-1} M S_{i+1}^{n}\right)_{\rho} \\
& =\frac{1}{n} \sum_{i=1}^{n} I\left(C_{i} S_{i} ; X_{i} Y_{i}\right)_{\rho} \\
& =I\left(C_{J} S_{J} ; X_{J} Y_{J} \mid J\right)_{\rho} \\
& =I\left(C_{J} S_{J} ; X_{J} J Y_{J}\right)_{\rho} \\
& =I(C S ; X Y)_{\rho}
\end{aligned}
$$

where the first equality follows from our definition of $X_{i}$ in (C33), the second holds since $J$ is a the classical variable with a uniform distribution over $\{1, \ldots, n\}$, the third because $I\left(C_{J} S_{J} ; J\right)_{\rho}=H\left(C_{J} S_{J}\right)_{\rho}-H\left(C_{J} S_{J} \mid J\right)_{\rho}=H(C S)_{\phi}-$ $H(C S)_{\phi}=0$, and the last equality follows from the definition of $C, S, X$, and $Y$ in (C36). This completes the proof of Theorem 3 .

[1] E. Bou-Harb, C. Fachkha, M. Pourzandi, M. Debbabi, and C. Assi, Communication security for smart grid distribution networks, IEEE Commun. Mag. 51, 42 (2013).

[2] J. Lopez, R. Rios, F. Bao, and G. Wang, Evolving privacy: From sensors to the internet of things, Future Generation Computer Systems 75, 46 (2017).

[3] R. Piqueras Jover and V. Marojevic, Security and protocol exploit analysis of the 5g specifications, IEEE Access 7, 24956 (2019).

[4] H. Wang, Q. Yang, Z. Ding, and H. V. Poor, Secure short-packet communications for mission-critical iot applications, IEEE Trans. Wireless Commun. 18, 2565 (2019).

[5] A. D. Wyner, The wire-tap channel, Bell Syst. Tech. J 54(8), 1355 (1975).

[6] N. Merhav and S. Shamai, Information rates subject to state masking, IEEE Trans. Inf. Theory 53, 2254 (2007).

[7] M. Naor, Bit commitment using pseudorandomness, J. Cryptology 4, 151 (1991).

[8] M. Naor and B. Pinkas, Efficient oblivious transfer protocols., in Proc. 12th Ann. ACM-SIAM Symp. on Discr. Algo. (SODA'2001), Vol. 1 (2001) pp. 448-457.

[9] C. S. Jensen, H. Lu, and M. L. Yiu, Location privacy techniques in client-server architectures, in Privacy in location-based applications (Springer, 2009) pp. 31-58.

[10] B. Semal, K. Markantonakis, and R. N. Akram, A rertificateless group authenticated key agreement protocol for secure communication in untrusted uav networks, in 2018 IEEE/AIAA 37th Dig. Avionics Syst. Conf. (DASC) (2018) pp. 1-8.

[11] P. Branco, L. Fiolhais, M. Goulão, P. Martins, P. Mateus, and L. Sousa, Roted: Random oblivious transfer for embedded devices, IACR Trans. Crypto. Hardw. Embedded Syst. , 215 (2021).

[12] G. P. Fettweis and H. Boche, 6G: The personal tactile internet - and open questions for information theory, IEEE BITS Info. Th. Mag. (2021).

[13] G. P. Fettweis and H. Boche, On 6G and trustworthiness, Communications of ACM, invited paper, to be published 2022..

[14] M. Le Treust and M. Bloch, Empirical coordination, state masking and state amplification: Core of the decoder's knowledge, in Proc. IEEE Int. Symp. Inf. Theory (ISIT'2016) (Barcelona, Spain, 2016) pp. 895-899. 
[15] M. Le Treust and M. R. Bloch, State leakage and coordination with causal state knowledge at the encoder, IEEE Trans. Inf. Theory 67, 805 (2021).

[16] O. O. Koyluoglu, R. Soundararajan, and S. Vishwanath, State amplification subject to masking constraints, IEEE Trans. Inf. Theory 62, 6233 (2016).

[17] O. O. Koyluoglu, R. Soundararajan, and S. Vishwanath, State amplification under masking constraints, in Proc. Allerton Conf. Commun., Control, Computing (Monticello, IL, USA, 2011) pp. 936-943.

[18] M. Dikshtein, A. Somekh-Baruch, and S. Shamai, Broadcasting information subject to state masking over a mimo state dependent gaussian channel, in Proc. IEEE Int. Symp. Inf. Theory (ISIT'2019) (Paris, France, 2019) pp. 275-279.

[19] S. Asoodeh, M. Diaz, F. Alajaji, and T. Linder, Information extraction under privacy constraints, Information 7, 15 (2016).

[20] K. Tutuncuoglu, O. Ozel, A. Yener, and S. Ulukus, State amplification and state masking for the binary energy harvesting channel, in Proc. IEEE Inf. Theory Workshop (ITW'2014) (Hobart, TAS, Australia, 2014) pp. 336-340.

[21] T. A. Courtade, Information masking and amplification: The source coding setting, in Proc. IEEE Int. Symp. Inf. Theory (ISIT'2012) (Cambridge, MA,USA, 2012) pp. 189-193.

[22] S. X. Ng, A. Conti, G. L. Long, P. Muller, A. Sayeed, J. Yuan, and L. Hanzo, Guest editorial advances in quantum communications, computing, cryptography, and sensing, IEEE J. Selected Areas in Commun. 38, 405 (2020).

[23] A. S. Holevo, The capacity of the quantum channel with general signal states, IEEE Trans. Inf. Theory 44, 269 (1998).

[24] B. Schumacher and M. D. Westmoreland, Sending classical information via noisy quantum channels, Phys. Rev. A 56, 131 (1997).

[25] P. W. Shor, Additivity of the classical capacity of entanglement-breaking quantum channels, J. Math. Phys. 43, 4334 (2002).

[26] A. S. Holevo, Quantum systems, channels, information: a mathematical introduction, Vol. 16 (Walter de Gruyter, 2012 ).

[27] I. Devetak, The private classical capacity and quantum capacity of a quantum channel, IEEE Trans. Inf. Theory 51, 44 (2005).

[28] R. Bassoli, R. Ferrara, S. Saeedinaeeni, C. Deppe, H. Boche, F. H. P. Fitzek, and G. Jansen, Quantum Communication Networks (Springer (in prep.), 2020).

[29] C. H. Bennett, P. W. Shor, J. A. Smolin, and A. V. Thapliyal, Entanglement-assisted capacity of a quantum channel and the reverse shannon theorem, IEEE Trans. Inf. Theory 48, 2637 (2002).

[30] H. Boche, N. Cai, and J. Nötzel, The classical-quantum channel with random state parameters known to the sender, J. Physics A: Math. and Theor. 49, 195302 (2016).

[31] U. Pereg, Communication over quantum channels with parameter estimation, in Proc. IEEE Int. Symp. Inf. Theory (ISIT'2020) (2020).

[32] U. Pereg, Communication over quantum channels with parameter estimation, IEEE Trans. Inf. Theory 68, 359 (2022).

[33] N. A. Warsi and J. P. Coon, Coding for classical-quantum channels with rate limited side information at the encoder: information-spectrum approach, IEEE Trans. Inf. Theory 63, 3322 (2017).

[34] F. Dupuis, Coding for quantum channels with side information at the transmitter, arXiv preprint arXiv:0805.3352 (2008).

[35] F. Dupuis, The capacity of quantum channels with side information at the transmitter, in Proc. IEEE Int. Symp. Inf. Theory (ISIT'2009) (2009) pp. 948-952.

[36] A. Anshu, R. Jain, and N. A. Warsi, On the near-optimality of one-shot classical communication over quantum channels, J. Math. Phys. 60, 012204 (2019).

[37] U. Pereg, Entanglement-assisted capacity of quantum channels with side information, in Int. Zürich Seminar Inf. Commun. (IZS'2020) (Zürich, Switzerland, 2020) pp. 106-110.

[38] U. Pereg, Entanglement-assisted capacity of quantum channels with side information, arXiv: 1909.09992 (2019).

[39] Z. Luo and I. Devetak, Channel simulation with quantum side information, IEEE Trans. Inf. Theory 55, 1331 (2009).

[40] A. Wyner and J. Ziv, The rate-distortion function for source coding with side information at the decoder, IEEE Trans. Inf. Theory 22, 1 (1976).

[41] I. Devetak and A. Winter, Classical data compression with quantum side information, Phys. Rev. A 68, 042301 (2003).

[42] J. T. Yard and I. Devetak, Optimal quantum source coding with quantum side information at the encoder and decoder, IEEE Trans. Inf. Theory 55, 5339 (2009).

[43] M. Hsieh and S. Watanabe, Channel simulation and coded source compression, IEEE Trans. Inf. Theory 62, 6609 (2016).

[44] N. Datta, C. Hirche, and A. Winter, Convexity and operational interpretation of the quantum information bottleneck function, in Proc. IEEE Int. Symp. Inf. Theory (ISIT'2019) (Paris, France, 2019) pp. 1157-1161.

[45] N. Datta, C. Hirche, and A. Winter, Convexity and operational interpretation of the quantum information bottleneck function, arXiv:1810.03644 (2018).

[46] H. C. Cheng, E. P. Hanson, N. Datta, and M. H. Hsieh, Duality between source coding with quantum side information and cq channel coding, in Proc. IEEE Int. Symp. Inf. Theory (ISIT'2019) (Paris, France, 2019) pp. 1142-1146.

[47] Z. Baghali Khanian and A. Winter, Distributed compression of correlated classical-quantum sources or: The price of ignorance, IEEE Trans. Inf. Theory 66, 5620 (2020).

[48] N. Cai, A. Winter, and R. W. Yeung, Quantum privacy and quantum wiretap channels, Probl. Info. Transm. 40, 318 (2004).

[49] M. H. Hsieh, Z. Luo, and T. Brun, Secret-key-assisted private classical communication capacity over quantum channels, Physical Review A 78, 042306 (2008).

[50] K. Li, A. Winter, X. Zou, and G. Guo, Private capacity of quantum channels is not additive, Physical Review Letters 103, 120501 (2009).

[51] M. M. Wilde, Comment on "secret-key-assisted private classical communication capacity over quantum channels", Phys. 
Rev. A 83, 046303 (2011).

[52] S. Watanabe, Private and quantum capacities of more capable and less noisy quantum channels, Phys. Rev. A 85, 012326 (2012).

[53] D. Elkouss and S. Strelchuk, Superadditivity of private information for any number of uses of the channel, Phys. Rev. Lett. 115, 040501 (2015).

[54] A. Anshu, M. Hayashi, and N. A. Warsi, Secure communication over fully quantum Gel'fand-Pinsker wiretap channel, in Proc. IEEE Int. Symp. Inf. Theory (ISIT'2018) (Vail, CO, USA, 2018) pp. 2679-2683.

[55] H. Boche, M. Cai, J. Nötzel, and C. Deppe, Secret message transmission over quantum channels under adversarial quantum noise: Secrecy capacity and super-activation, J. Math. Phys. 60, 062202 (2019).

[56] H. Boche, M. Cai, C. Deppe, and J. Nötzel, Classical-quantum arbitrarily varying wiretap channel: Common randomness assisted code and continuity, Quantum Info. Proc. 16, 35 (2017).

[57] R. König, R. Renner, A. Bariska, and U. Maurer, Small accessible quantum information does not imply security, Physical Review Letters 98, 140502 (2007).

[58] S. Guha, P. Hayden, H. Krovi, S. Lloyd, C. Lupo, J. H. Shapiro, M. Takeoka, and M. M. Wilde, Quantum enigma machines and the locking capacity of a quantum channel, Physical Review X 4, 011016 (2014).

[59] C. Lupo, M. M. Wilde, and S. Lloyd, Quantum data hiding in the presence of noise, IEEE Trans. Inf. Theory 62, 3745 (2016).

[60] F. Salek, M. Hsieh, and J. R. Fonollosa, Publicness, privacy and confidentiality in the single-serving quantum broadcast channel, in Proc. IEEE Int. Symp. Inf. Theory (ISIT'2019) (Paris, France, 2019) pp. 1712-1716.

[61] H. Aghaee and B. Akhbari, Classical-quantum multiple access wiretap channel, in Int'l ISC Conf. Info. Secur. Crypt. (ISCISC'2019) (Mashhad, Iran, 2019).

[62] H. Boche, G. Janßen, and S. Saeedinaeeni, Universal superposition codes: Capacity regions of compound quantum broadcast channel with confidential messages, J. Math. Phys. 61, 042204 (2020).

[63] U. Pereg, C. Deppe, and H. Boche, Quantum channel state masking, IEEE Trans. Inf. Theory 67, 2245 (2021).

[64] P. Hayden, M. Horodecki, A. Winter, and J. Yard, A decoupling approach to the quantum capacity, Open Sys. Inf. Dynamics 15, 7 (2008).

[65] M. Hsieh, I. Devetak, and A. Winter, Entanglement-assisted capacity of quantum multiple-access channels, IEEE Trans. Inf. Theory 54, 3078 (2008).

[66] M. M. Wilde, Quantum information theory, 2nd ed. (Cambridge University Press, 2017).

[67] I. Devetak and P. W. Shor, The capacity of a quantum channel for simultaneous transmission of classical and quantum information, Commun. in Math. Phys. 256, 287 (2005).

[68] C. H. Bennett, D. P. DiVincenzo, and J. A. Smolin, Capacities of quantum erasure channels, Phys. Rev. Lett. 78, 3217 (1997).

[69] H. G. Eggleston, Convexity, J. London Math. Society 1, 183 (1966).

[70] I. Csiszár and J. Körner, Information Theory: Coding Theorems for Discrete Memoryless Systems, 2nd ed. (Cambridge University Press, 2011).

[71] A. El Gamal and Y. Kim, Network Information Theory (Cambridge University Press, 2011).

[72] B. Schumacher, Quantum coding, Phys. Rev. A 51, 2738 (1995).

[73] M. A. Nielsen and I. Chuang, Quantum computation and quantum information (2010).

[74] A. Winter, Tight uniform continuity bounds for quantum entropies: conditional entropy, relative entropy distance and energy constraints, Commun. in Math. Phys. 347, 291 (2016). 